\title{
Pacific
}

Journal of

Mathematics

\section{STRONG SUBMODULES \\ OF ALMOST PROJECTIVE MODULES}

GÁBOR BRAUN AND JAN TRLIFAJ 


\title{
STRONG SUBMODULES OF ALMOST PROJECTIVE MODULES
}

\author{
GÁBOR BRAUN AND JAN TRLIFAJ
}

\begin{abstract}
The structure of almost projective modules can be better understood in the case when the following Condition (P) holds: The union of each countable pure chain of projective modules is projective. We prove this condition, and its generalization to pure-projective modules, for all countable rings, using the new notion of a strong submodule of the union.

However, we also show that Condition (P) fails for all Prüfer domains of finite character with uncountable spectrum, and in particular, for the polynomial ring $K[x]$, where $K$ is an uncountable field. One can even prescribe the $\Gamma$-invariant of the union. Our results generalize earlier work of Hill, and complement recent papers by Macías-Díaz, Fuchs, and Rangaswamy.
\end{abstract}

By a classic theorem of Kaplansky, the structure theory of projective modules over an arbitrary ring reduces to that of countably generated ones. In stark contrast, almost projective modules (modules possessing a rich supply of small projective submodules) generally have a very complex structure. Perhaps the most successful invariant measuring their complexity is the $\Gamma$-invariant. A projective module has a trivial $\Gamma$-invariant [Eklof 1993; Eklof and Mekler 2002].

There are additional conditions on almost projective modules that guarantee projectivity. In his work on Whitehead groups, Hill [1970] discovered a remarkable condition in the particular case of abelian groups: if $A$ is the union of a countable pure chain of (arbitrarily large) projective groups, then $A$ is projective. Here, we call the analogous property for modules over an arbitrary ring Condition $(P)$.

In the past decade, several authors have attempted to extend Hill's result and establish Condition (P) for large classes of rings, notably for commutative domains and noetherian rings [Fuchs and Rangaswamy 2011; Fuchs and Salce 2001]. So far, Macías-Díaz [2010] has obtained the strongest result, that Prüfer domains with countable spectrum have Condition $(\mathrm{P})$.

Section 1 of our paper gives more motivation for considering Condition (P), by showing its role in relating various notions of almost projectivity appearing in the

Research partially supported by the Hungarian Scientific Research Fund, Grant NK 81203. Research supported by the PPP project MEB 101005, GAČR 201/09/0816, and MSM 0021620839.

MSC2010: primary 13C10, 16D40; secondary 03E75, 13F05, 13G05, $16 \mathrm{P} 70$.

Keywords: Almost projective module, pure chain, strong submodule, $\Gamma$-invariant, Prüfer domain. 
literature. In Section 2, we prove Condition (P) and some of its generalizations for all countable rings, using the new notion of a strong submodule.

However, in Section 3, we show that Condition (P) fails completely for all Prüfer domains of finite character with uncountable spectrum (and thus, for example, for the polynomial ring $K[x]$, where $K$ is any uncountable field). Here, "completely" refers to the fact that there are essentially no restrictions on the $\Gamma$-invariant of $A$.

In what follows, $R$ denotes a ring (that is, an associative ring with 1 ), and the term module means a right $R$-module.

\section{Almost projective modules}

The following definition is the analogue of [Eklof and Mekler 2002, IV.1.1] for general rings, with "free" replaced by "projective".

Definition 1.1. Let $R$ be a ring and $\kappa$ a regular uncountable cardinal. A module $M$ is called $\kappa$-projective if there exists a set $\mathscr{S}$ consisting of $<\kappa$-generated projective submodules of $M$ such that

(i) each subset of $M$ of cardinality $<\kappa$ is contained in an element of $\mathscr{Y}$, and

(ii) $\mathscr{Y}$ is closed under unions of well-ordered chains of length $<\kappa$.

We recall some other relevant notions for the study of almost projectivity (see, for example, [Eklof and Mekler 2002, IV.1; Trlifaj 1995]).

Definition 1.2. Let $R$ be a ring and $\kappa$ a regular uncountable cardinal. A module $M$ is called weakly $\kappa$-projective if each subset of $M$ of cardinality $<\kappa$ is contained in a pure submodule $N$ of $M$ that is $<\kappa$-generated and projective.

Recall that a module $M$ is flat if the functor $M \otimes_{R}-$ is exact, and that $M$ is Mittag-Leffler if the canonical map

$$
M \otimes_{R} \prod_{i \in I} Q_{i} \rightarrow \prod_{i \in I}\left(M \otimes_{R} Q_{i}\right)
$$

is monic for each family of left $R$-modules $\left(Q_{i} \mid i \in I\right)$.

Lemma 1.3 [Raynaud and Gruson 1971; Herbera and Trlifaj 2009]. Let $R$ be a ring and $M$ a module. Then the following conditions are equivalent:

(i) $M$ is $\aleph_{1}$-projective.

(ii) $M$ is weakly $\aleph_{1}$-projective.

(iii) Each finite subset of $M$ is contained in a projective, countably generated and pure submodule of $M$.

(iv) $M$ is flat Mittag-Leffler. 
Also, if $\kappa$ is a regular uncountable cardinal and $M$ is $\kappa$-projective, then $M$ is $\aleph_{1^{-}}$ projective.

Proof. The equivalence of (i), (ii) and (iii) is proved in [Raynaud and Gruson 1971] (see also [Drinfeld 2006]), while (i) and (iv) are equivalent by [Herbera and Trlifaj 2009, Theorem 2.9(i)] (see also [Rothmaler 1994; 1997]). The last statement is [Herbera and Trlifaj 2009, Theorem 2.9(ii)].

The implication (i) $\Rightarrow$ (ii) extends to arbitrary regular uncountable cardinals $\kappa$ :

Lemma 1.4. Let $R$ be a ring, $M$ a module, and $\kappa$ an infinite cardinal.

(i) Assume that $M$ is $\aleph_{1}$-projective. Then each subset of $M$ of cardinality $\leq \kappa$ is contained in a $\leq \kappa$-generated pure submodule of $M$.

(ii) Assume that $\kappa$ is regular uncountable and $M$ is $\kappa$-projective. Then $M$ is weakly $\kappa$-projective.

Proof. (i) We prove the claim by induction on $\kappa$. The case of $\kappa=\aleph_{0}$ follows by Lemma 1.3.

Assume $\kappa \geq \aleph_{1}$, and let $X=\left\{x_{\alpha} \mid \alpha<\kappa\right\}$ be a subset of $M$ of cardinality $\kappa$. For each $\alpha<\kappa$, let $X_{\alpha}=\left\{x_{\beta} \mid \beta<\alpha\right\}$. By induction on $\alpha$, we define an increasing chain $\left(P_{\alpha} \mid \alpha<\kappa\right)$ of $<\kappa$-generated pure submodules of $M$ as follows: $P_{0}=0$, $P_{\alpha+1}$ is a $<\kappa$-generated pure submodule of $M$ containing $X_{\alpha} \cup P_{\alpha}$ (which exists by the inductive premise), and $P_{\alpha}=\bigcup_{\beta<\alpha} P_{\beta}$ when $\alpha<\kappa$ is a limit ordinal. Then $P=\bigcup_{\alpha<\kappa} P_{\alpha}$ is a $\leq \kappa$-generated pure submodule of $M$ containing $X$.

(ii) Let $\mathscr{S}$ be as in Definition 1.1, and let $X$ be a subset of $M$ of cardinality $<\kappa$. By condition (i) of Definition 1.1, $X$ is contained in a $<\kappa$-generated projective submodule $P_{0} \in \mathscr{Y}$. By the last statement of Lemma 1.3 and by Lemma 1.4(i), $P_{0}$ is contained in a $<\kappa$-generated pure submodule $Q_{0}$ of $M$. Proceeding similarly, we obtain a countable chain

$$
P_{0} \subseteq Q_{0} \subseteq P_{1} \subseteq Q_{1} \subseteq \cdots \subseteq P_{n} \subseteq Q_{n} \subseteq \cdots,
$$

where $P_{n} \in \mathscr{Y}$, so $P_{n}$ is $<\kappa$-generated and projective, and $Q_{n}$ is $<\kappa$-generated and pure in $M$, for all $n<\omega$. Let $P=\bigcup_{n<\omega} P_{n}=\bigcup_{n<\omega} Q_{n}$. Then $P \in \mathscr{S}$ by condition (ii) of Definition 1.1, and $P$ is pure in $M$.

Whatever the cardinality of the ring $R$, Lemma 1.4(i) makes it possible to purify a submodule without increasing the number of generators. So in the particular case when $R$ is a right hereditary ring, $\kappa$-projectivity and weak $\kappa$-projectivity are equivalent (to the property that each $<\kappa$-generated submodule is projective). However, the converse of Lemma 1.4(ii) fails in general:

Example 1.5. Let $\kappa>\aleph_{1}$ be a regular cardinal, let $K$ be a field, and let $R$ denote the endomorphism ring of a $\kappa$-dimensional $K$-linear space modulo its maximal 
ideal. Then there exists a $\kappa$-generated right ideal $I$ in $R$ such that $I$ is weakly $\kappa$-projective, but not $\kappa$-projective [Trlifaj 1995, Theorem 8].

Another relevant property is the following (where a chain $\left(P_{n} \mid n<\omega\right)$ is a pure chain if $P_{n}$ is a pure submodule of $P_{n+1}$ for each $\left.n<\omega\right)$ :

Definition 1.6. Let $R$ be a ring. Then $R$ satisfies Condition $(P)$ if for each pure chain $\left(P_{n} \mid n<\omega\right)$ consisting of projective modules, the module $P=\bigcup_{n<\omega} P_{n}$ is projective.

Condition (P) yields a characterization of weak $\kappa$-projectivity:

Proposition 1.7. Let $R$ be a ring satisfying Condition $(P)$. Let $M$ be a module and $\kappa$ a regular infinite cardinal. Then $M$ is weakly $\kappa$-projective if and only if there exists a set $\mathscr{Y}$ consisting of $<\kappa$-generated projective submodules of $M$ such that

(i) each subset of $M$ of cardinality $<\kappa$ is contained in an element of $\mathscr{Y}$, and

(ii) $\mathscr{Y}$ is closed under unions of countable chains.

Proof. By [Herbera and Trlifaj 2009, Corollary 2.3], assumptions (i) and (ii) assure $\aleph_{1}$-projectivity of $M$, so the "if" implication is proved as in Lemma 1.4. For the "only if", let $\mathscr{Y}$ be the set of all $<\kappa$-generated projective and pure submodules of $M$. Then (i) holds by the assumption. If $M_{0} \subseteq \cdots \subseteq M_{n} \subseteq M_{n+1} \subseteq \cdots$ is a countable chain of elements of $\mathscr{Y}$, then $M_{\omega}=\bigcup_{n<\omega} M_{n}$ is projective by Condition (P), so $M_{\omega} \in \mathscr{Y}$.

Condition (P) holds for $R=\mathbb{Z}$. This was shown by Hill [1970], who proved thus the singular compactness of almost free abelian groups of cardinality $\aleph_{\alpha}$, where $\alpha$ has cofinality $\omega$.

More generally, Condition $(\mathrm{P})$ is known to hold for all Prüfer domains with countably many maximal ideals [Macías-Díaz 2010, Corollary 15], and hence for all valuation domains. In Theorem 2.5 below, we prove it for all countable rings.

However, attempts to prove Condition $(\mathrm{P})$ for arbitrary domains in [Fuchs and Salce 2001, XVI.1.4] and [Fuchs and Rangaswamy 2011, Theorem 1.3] have gaps; in fact, as we see in Theorem 3.1, Condition (P) fails even for $R=K[x]$, where $K$ is an uncountable field.

The main goal of the next section is to prove Condition $(\mathrm{P})$, and hence the equivalence in Proposition 1.7, for all countable rings. Before proceeding to that point, we note that under additional assumptions on $R$ and $M$, the equivalence holds even without assuming Condition (P):

Proposition 1.8. Let $\kappa$ be an infinite cardinal, and let $R$ be a ring that is either a domain or is right $<\kappa$-noetherian (that is, every right ideal is $<\kappa$-generated). Let $M$ be a module of projective dimension $\leq 1$. Then $M$ is weakly $\kappa$-projective if and only if there exists a set $\mathscr{Y}$ consisting of $<\kappa$-generated projective submodules of $M$ such that 
(i) each subset of $M$ of cardinality $<\kappa$ is contained in an element of $\mathscr{Y}$, and

(ii) $\mathscr{S}$ is closed under unions of countable chains.

The following lemma helps in finding projective submodules:

Lemma 1.9. Let $M$ be a module of projective dimension at most 1 . Let $N$ be a tight submodule; that is, let $M / N$ have also projective dimension at most 1 . If $N$ is contained in a projective submodule of $M$, then $N$ is projective.

Proof. Let $P$ be a projective module such that $N \subseteq P \subseteq M$. We can estimate the projective dimensions of various modules built from $N, P$ and $M$ using the long exact sequence for Ext as follows:

$$
\begin{aligned}
\text { proj.dim } M / P & \leq \max \{\text { proj.dim } M, \text { proj.dim } P+1\} \leq 2, \\
\text { proj.dim } P / N & \leq \max \{\text { proj.dim } M / N, \text { proj.dim } M / P-1\} \leq 1, \\
\text { proj.dim } N & \leq \max \{\text { proj.dim } P, \text { proj.dim } P / N-1\} \leq 0 .
\end{aligned}
$$

The last line shows that $N$ is projective.

Proof of Proposition 1.8. As in the proof of Proposition 1.7, the conditions (i) and (ii) of Proposition 1.8 imply that $M$ is $\kappa$-projective (because neither Condition (P) nor any of our additional assumptions are needed there).

For the other direction, we note that by the assumptions on $R$, there is a Hill family consisting of tight submodules of $M$ : when $R$ is a domain, this follows by [Fuchs and Salce 2001, Proposition VI.5.1] and [Göbel and Trlifaj 2006, 4.2.6], and when $R$ is $<\kappa$-noetherian, we apply [Göbel and Trlifaj 2006, 4.1.11 and 4.2.6].

Let $\mathscr{S}$ be the subfamily of the $<\kappa$-generated members of this family. Conditions (i) and (ii) automatically hold. Finally, the assumption of weak $\kappa$-projectivity and Lemma 1.9 imply that $\mathscr{S}$ consists of projective modules.

\section{Hill families of strong submodules}

We start this section by considering a general version of Condition $(\mathrm{P})$, where the chain $\left(P_{n} \mid n<\omega\right)$ is not necessarily pure, and the modules $P_{n}(n<\omega)$ are direct sums of modules from a given class $\mathscr{C}$ consisting of countably presented modules or modules of countable rank. The relevant notion here is that of a strong submodule. It is introduced in the following definition, where, for a class of modules $\mathscr{C}$, we denote by Sum $(\mathscr{C})$ the class of all direct sums of copies of modules from $\mathscr{C}$.

Definition 2.1. Let $R$ be a ring and $\mathscr{C}$ a class of modules.

Let $\left(P_{n} \mid n<\omega\right)$ be a countable increasing chain of modules, and suppose $P=\bigcup_{n<\omega} P_{n}$. Assume that $P_{n} \in \operatorname{Sum}(\mathscr{C})$ for each $n<\omega$; that is, there exists a decomposition $P_{n}=\bigoplus_{\alpha<\kappa_{n}} P_{n, \alpha}$, where $P_{n, \alpha}$ is isomorphic to an element of $\mathscr{C}$ for each $\alpha<\kappa_{n}$. 
We fix these decompositions, and for each $n<\omega$ and each subset $S \subseteq \kappa_{n}$, define $P(n, S)=\bigoplus_{\alpha \in S} P_{n, \alpha}$. So, in particular, $P_{n}=P\left(n, \kappa_{n}\right)$.

A submodule $N$ of $P$ is called strong if there exist $\left(A_{n} \mid n<\omega\right)$ such that $A_{n} \subseteq \kappa_{n}$ and $N \cap P_{n}=P\left(n, A_{n}\right)$ for each $n<\omega$. The sequence $\left(A_{n} \mid n<\omega\right)$ is then uniquely determined by $N$; it is the witnessing sequence for $N$.

In this section, $P$ denotes the union $\bigcup_{n<\omega} P_{n}$, where $\left(P_{n} \mid n<\omega\right)$ is a countable increasing chain of modules, as in Definition 2.1.

In the case when $\mathscr{C}$ is the class of all countably presented projective modules, Definition 2.1 covers the setting of Condition (P), because by a classic theorem of Kaplansky, each projective module is a direct sum of modules in $\mathscr{C}$.

Note that 0 and $P$ are strong submodules of $P$. Also, unions of chains of strong submodules are strong, and so are arbitrary intersections of strong submodules. Indeed, in Theorem 2.9, we prove that strong submodules are abundant.

If $N$ is strong in $P$ and the chain $\left(P_{n} \mid n<\omega\right)$ is pure, then $N$ is a pure submodule of $P$, because $N=\bigcup_{n<\omega} N \cap P_{n}$ and $N \cap P_{n}$ is a direct summand in the pure submodule $P_{n}$ of $P$ for each $n<\omega$.

For the next lemma, we recall that a ring $R$ is right $\aleph_{0}$-noetherian provided that each right ideal of $R$ is countably generated. For example, all right noetherian rings, and all countable rings, are right $\aleph_{0}$-noetherian. It is easy to see that a ring $R$ is right $\aleph_{0}$-noetherian if and only if each submodule of a countably generated module is countably generated.

Lemma 2.2. Assume that $R$ is right $\aleph_{0}$-noetherian and $\mathscr{b}$ consists of countably presented modules, or that $R$ is a commutative domain and $\mathscr{C}$ consists of torsionfree modules of countable rank, respectively. Let $N$ be a strong submodule of $P$ with witnessing sequence $\left(A_{n} \mid n<\omega\right)$. Let $C$ be a countable subset of $P$ or a subset of $P$ such that $\langle C\rangle$ has countable rank, respectively.

Then there is a strong submodule $N^{\prime}$ of $P$ such that $N \cup C \subseteq N^{\prime}$, the witnessing sequence $\left(A_{n}^{\prime} \mid n<\omega\right)$ for $N^{\prime}$ satisfies $A_{n} \subseteq A_{n}^{\prime}$, and $A_{n}^{\prime} \backslash A_{n}$ is countable for each $n<\omega$.

Proof. We simultaneously and recursively construct chains $\left(C_{n, i}: i<\omega\right)$ of subsets of $\kappa_{n}$.

As a start, for each $n<\omega$, put $A_{n} \subseteq C_{n, 0} \subseteq \kappa_{n}$, with $C_{n, 0} \backslash A_{n}$ countable and $C \cap P_{n} \subseteq P\left(n, C_{n, 0}\right)$.

For $i \geq 0$, let $C_{n, i} \subseteq C_{n, i+1} \subseteq \kappa_{n}$, with $C_{n, i+1} \backslash C_{n, i}$ countable and

$$
P\left(m, C_{m, i}\right) \cap P_{n} \subseteq P\left(n, C_{n, i+1}\right)
$$

for all $m$.

Finally, we define $A_{n}^{\prime}=\bigcup_{i<\omega} C_{n, i}$ for each $n<\omega$. Then $A_{n} \subseteq A_{n}^{\prime} \subseteq \kappa_{n}$, and $A_{n}^{\prime} \backslash A_{n}$ is countable for each $n<\omega$. Let $N^{\prime}=\bigcup_{n<\omega} P\left(n, A_{n}^{\prime}\right)=\bigcup_{n, i<\omega} P\left(n, C_{n, i}\right)$. 
The $P\left(n, C_{n, i}\right)$ form an upper directed system of submodules, so their union is a submodule.

Recall that $P\left(m, C_{m, i}\right) \cap P_{n} \subseteq P\left(n, C_{n, i+1}\right)$ for all $m, n, i<\omega$, and hence $N^{\prime} \cap$ $P_{n}=P\left(n, A_{n}^{\prime}\right)$. All in all, $N^{\prime}$ is a strong submodule of $P$ with witnessing sequence $\left(A_{n}^{\prime} \mid n<\omega\right)$.

Since $C \cap P_{n} \subseteq P\left(n, A_{n}^{\prime}\right)$ for each $n<\omega$, we conclude that $N \cup C \subseteq N^{\prime}$.

Lemma 2.2 serves as inductive step for proving the following:

Proposition 2.3. Assume either that $R$ is right $\aleph_{0}$-noetherian and $\mathscr{C}$ consists of countably presented modules, or that $R$ is a commutative domain and $\mathscr{C}$ consists of torsion-free modules of countable rank, respectively.

Then $P$ is the union of a continuous increasing chain $M=\left(M_{\alpha} \mid \alpha<\lambda\right)$ of strong submodules of $P$, such that for each $\alpha<\lambda$, there is a countably generated or countable-rank submodule $N_{\alpha}$ of $P$, respectively, with $M_{\alpha+1}=M_{\alpha}+N_{\alpha}$.

Proof. Let $\left\{p_{\alpha} \mid \alpha<\lambda\right\}$ be an $R$-generating subset of $P$. Since $M_{0}=0$ is strong, and the union of a chain of strong submodules is strong, it remains to perform the nonlimit step of the construction. However, applying Lemma 2.2 for $N=M_{\alpha}$ and $C=\left\{p_{\alpha}\right\}$, we can take $N_{\alpha}=\sum_{n<\omega} P\left(n, A_{n}^{\prime} \backslash A_{n}\right)$ and $M_{\alpha+1}=N^{\prime}$.

We can prove more in the particular case of countable rings. We consider a class of modules $\mathscr{b}$ to have Property $(C)$ if for each increasing pure chain of modules $\left(Q_{n} \mid n<\omega\right)$ such that $Q_{n} \in \operatorname{Sum}(\mathscr{C})$ for all $n<\omega$, and each countably presented pure submodule $C$ of $\bigcup_{n<\omega} Q_{n}$, the module $C$ is $\mathscr{b}$-filtered. Also, $\mathscr{C}$ has Property $(C+)$ if the same assumptions yield the stronger conclusion of $C \in \operatorname{Sum}(\mathscr{C})$.

For example, the class of all countably presented modules and the class of all projective modules have Property $(\mathrm{C}+)$, because the union of a pure chain of projective modules is always $\aleph_{1}$-projective, by Lemma 1.3.

Lemma 2.4. Let $R$ be a countable ring. Let $\mathscr{C}$ be a class of countably presented modules that has Property $(C)$. Let $\left(P_{n} \mid n<\omega\right)$ be an increasing pure chain of modules such that $P_{n} \in \operatorname{Sum}(\mathscr{C})$ for all $n<\omega$, and let $P=\bigcup_{n<\omega} P_{n}$. Then $P$ is C-filtered.

Also, if $\mathscr{C}$ has Property $(C+)$, then $P$ is the union of a continuous increasing chain $M=\left(M_{\alpha} \mid \alpha<\lambda\right)$ consisting of strong submodules of $P$ such that $M_{\alpha+1} / M_{\alpha} \in$ $\operatorname{Sum}(\mathscr{b})$.

Proof. Let $\left(P_{n} \mid n<\omega\right)$ be an increasing pure chain of modules with $P_{n} \in \operatorname{Sum}$ ('C) for all $n<\omega$. Since $R$ is countable, the continuous chain $M$ from Proposition 2.3 can be taken with the additional property of $M_{\alpha}+P_{n}$ being pure in $P$ for all $n<\omega$ and $\alpha<\kappa$. This is arranged by improving Lemma 2.2 for countable $R$ : when for the strong submodule $N$, all the submodules $N+P_{n}$ are pure, then $N^{\prime}$ can be chosen with the $N^{\prime}+P_{n}$ also pure. 
It follows that for each $\alpha<\kappa$, the factor $Q=P / M_{\alpha}$ is the union of the pure chain $\left(Q_{n} \mid n<\omega\right)$, where $Q_{n}=\left(M_{\alpha}+P_{n}\right) / M_{\alpha}$. Also, $Q_{n} \cong P_{n} /\left(P_{n} \cap M_{\alpha}\right) \in$ Sum (C) , because $M_{\alpha}$ is strong. Similarly, the countably presented submodule $C=M_{\alpha+1} / M_{\alpha}$ is pure in $Q$, so $C$ is $\mathscr{C}$-filtered by Property (C). Then $P=\bigcup_{\alpha<\kappa} M_{\alpha}$ is $\mathscr{b}$-filtered as well.

Also, if $\mathscr{C}$ has Property $(\mathrm{C}+)$, then $C=M_{\alpha+1} / M_{\alpha} \in \operatorname{Sum}(\mathscr{C})$.

The assumptions of Lemma 2.4 are satisfied for $R$ countable and $\mathscr{C}$ the class of all countably generated projective modules. Since in this case $\mathscr{C}$-filtered is the same as projective, we get:

Theorem 2.5. Let $R$ be a countable ring. Then $R$ satisfies Condition $(P)$.

As another consequence, we obtain the general version of Condition $(\mathrm{P})$ for the case when $R$ is countable, $\mathscr{C}$ has Property $(\mathrm{C}+)$, and $\mathscr{C}$ consists of finitely presented modules:

Corollary 2.6. Let $R$ be a countable ring, and let $\mathscr{C}$ be a class of finitely presented modules that has Property $(C+)$. Let $\left(P_{n} \mid n<\omega\right)$ be an increasing pure chain of modules such that $P_{n} \in \operatorname{Sum}(\mathscr{C})$ for all $n<\omega$ and that $P=\bigcup_{n<\omega} P_{n}$. Then $P \in \operatorname{Sum}(\mathfrak{b})$.

Proof. By Lemma 2.4, $P$ is the union of a continuous increasing chain

$$
M=\left(M_{\alpha} \mid \alpha<\lambda\right),
$$

consisting of strong submodules of $P$ such that $M_{\alpha+1} / M_{\alpha} \in$ Sum (' $\left.\mathscr{C}\right)$. In particular, $M_{\alpha}$ is pure in $M_{\alpha+1}$ for each $n<\omega$. As $\mathscr{C}$ consists of finitely presented modules, $M_{\alpha+1} / M_{\alpha}$ is pure-projective, and the embedding $M_{\alpha} \hookrightarrow M_{\alpha+1}$ splits. This proves that $P \in \operatorname{Sum}(\mathscr{b})$.

A variation of Corollary 2.6 gives the version of Condition $(\mathrm{P})$ for pure-projective modules over countable rings.

Theorem 2.7. Let $R$ be a countable ring, $\left(P_{n} \mid n<\omega\right)$ be an increasing pure chain of pure-projective modules, and $P=\bigcup_{n<\omega} P_{n}$. Then $P$ is pure-projective.

Proof. By [Raynaud and Gruson 1971, Seconde partie, Corollaire 2.2.2], a countably presented module is pure-projective if and only if it is Mittag-Leffler, and the latter property is clearly inherited by pure submodules. As in Lemma 2.4, we infer that $P$ is the union of a continuous chain $M$ consisting of strong submodules of $P$ such that all consecutive factors in $M$ are pure-projective, and hence $P$ is pureprojective as well.

Alternatively, we can deduce Theorem 2.5 from Theorem 2.7, because projective $=$ flat + pure-projective. 
Of course, the union of a nonpure countable chain of projective modules need not be projective even for countable rings: just consider $R=\mathbb{Z}$ and $\mathbb{Q}$ as the union of the chain of free groups $(1 / n ! \cdot \mathbb{Z} \mid n<\omega)$.

Also, the general version of Condition $(\mathrm{P})$ for pure chains consisting of modules from Sum $(\mathscr{C})$ may fail even for countable rings and $\mathscr{C}$ having Property (C). That is, even though $P$ is $\mathscr{C}$-filtered by Lemma $2.4, P \notin \operatorname{Sum}(\mathscr{C})$ in general:

Example 2.8. Let $R$ be a simple, countable von Neumann regular ring that is not artinian - for example, let $R$ be the directed union of the full matrix rings $M_{2^{n}}(\mathbb{Q})$ $(n<\omega)$ with the block diagonal embeddings

$$
\mathbb{Q} \subseteq M_{2}(\mathbb{Q}) \subseteq M_{4}(\mathbb{Q}) \subseteq \cdots \subseteq M_{2^{n}}(\mathbb{Q}) \subseteq M_{2^{n+1}}(\mathbb{Q}) \subseteq \cdots
$$

Consider a simple nonprojective module $S$, and let $\mathscr{C}$ be the class of all finitely $\{S\}$-filtered modules. Then $\mathscr{C}$ is a class of countable modules and has Property (C).

Define a chain of finite length modules $\left(P_{n} \mid n<\omega\right)$ such that $P_{0}=S$ and that $P_{n+1}$ fits in a nonsplit short exact sequence $0 \rightarrow P_{n} \subseteq P_{n+1} \rightarrow S \rightarrow 0$ for each $n<\omega$. This is possible by [Trlifaj 1996, Proposition 3.3]. This chain is pure because $R$ is von Neumann regular, so all $R$-modules are flat.

Let $P=\bigcup_{n<\omega} P_{n}$. Then $P_{n} \in \mathscr{C}$ for all $n<\omega$, and $P$ is $\mathscr{b}$-filtered, but $P \notin$ Sum (C) . Indeed, $S=P_{0}$ is an essential submodule of $P$, so $P$ is uniform, and hence indecomposable.

Returning to the general setting and using an idea by Hill, we can extend the chain $M$ from Proposition 2.3 further, to a large family of strong submodules:

Theorem 2.9. Assume that $R$ is right $\aleph_{0}$-noetherian and $\mathscr{C}$ consists of countably presented modules, or that $R$ is a commutative domain and $\mathscr{C}$ consists of torsionfree modules of countable rank, respectively. Let $\mathcal{M}=\left(M_{\alpha}: \alpha<\lambda\right)$ be a continuous increasing chain of strong submodules of $P$ as in Proposition 2.3. There is a family H of strong submodules of $P$ such that:

(i) $\mathcal{M} \subseteq \mathscr{H}$.

(ii) H is closed under arbitrary sums and intersections; in fact, H is a complete distributive sublattice of the modular lattice of all submodules of $P$.

(iii) Let $N, N^{\prime} \in \mathcal{H}$ be such that $N \subseteq N^{\prime}$. Then there exists a continuous increasing chain $\left(N_{\beta} \mid \beta \leq \tau\right)$ consisting of elements of $\mathcal{H}$ such that $\tau \leq \lambda, N_{0}=N$, $N_{\tau}=N^{\prime}$, and for each $\beta<\tau$ there is $\alpha<\kappa$ such that $N_{\beta+1} / N_{\beta}$ is isomorphic to $M_{\alpha+1} / M_{\alpha}$.

(iv) Let $N \in \mathscr{H}$, and let $X$ be a countable subset of $P$ (a subset of $P$ such that $\langle X\rangle$ has countable rank, respectively). Then there are $N^{\prime} \in \mathcal{H}$ and a submodule $Y \subseteq P$ such that $Y$ is countably generated (of countable rank, respectively) and $N \cup X \subseteq N^{\prime}=N+Y$. 
Proof. For all $\alpha<\lambda$ and $n<\omega$, let $D_{\alpha, n}=A_{\alpha+1, n} \backslash A_{\alpha, n}$, where $\left(A_{\alpha, n} \mid n<\omega\right)$ and $\left(A_{\alpha+1, n} \mid n<\omega\right)$ are the witnessing sequences for $M_{\alpha}$ and $M_{\alpha+1}$. By the assumption on the chain $\mathcal{M}$, all the sets $D_{\alpha, n}$ are countable. Let $\Delta_{\alpha}=\sum_{n<\omega} P\left(n, D_{\alpha, n}\right)$. Then $\Delta_{\alpha}$ is countably generated, and $M_{\alpha+1}=M_{\alpha}+\Delta_{\alpha}$ for each $\alpha<\lambda$.

As in [Göbel and Trlifaj 2006, §4.2], we call a subset $S$ of $\sigma$ closed when

$$
\Delta_{\alpha} \cap M_{\alpha} \subseteq \sum_{\beta<\alpha, \beta \in S} \Delta_{\beta}
$$

for each $\alpha \in S$. We define $\mathscr{H}=\left\{\sum_{\alpha \in S} \Delta_{\alpha} \mid S\right.$ is closed in $\left.\lambda\right\}$.

Since each ordinal $\sigma \leq \lambda$ is closed, $\mathcal{M} \subseteq \mathcal{H}$, and (i) holds. Properties (ii) and (iii) are proved in [Göbel and Trlifaj 2006, 4.2.6]. If $R$ is $\aleph_{0}$-noetherian, then (iv) is proved in [Göbel and Trlifaj 2006, 4.2.6], while in the domain case, (iv) follows by [Göbel and Trlifaj 2006, 4.2.8].

It remains to show that all modules in $\mathscr{H}$ are strong. Let $S$ be a closed subset of $\lambda$, and let $N=\sum_{\alpha \in S} \Delta_{\alpha}$ and $B_{n}=\bigcup_{\alpha \in S} D_{\alpha, n}$. It suffices to prove that $N \cap P_{n}=$ $P\left(n, B_{n}\right)$ for each $n<\omega$. The inclusion $\supseteq$ is clear from the definitions above.

Assume there exists $x \in\left(N \cap P_{n}\right) \backslash P\left(n, B_{n}\right)$. Then there is one of the form $x=x_{\alpha_{1}}+\cdots+x_{\alpha_{i}}$, where $\alpha_{1}<\cdots<\alpha_{i}$ are elements of $S$, and $x_{\alpha_{k}} \in \Delta_{\alpha_{k}} \backslash P\left(n, B_{n}\right)$ for all $1 \leq k \leq i$. Without loss of generality, we can assume that $\alpha=\alpha_{i}$ is minimal. Since $x \in M_{\alpha+1} \cap P_{n}=P\left(n, A_{\alpha+1, n}\right)$, we also have $x=y_{\beta_{1}}+\cdots+y_{\beta_{j}}$, where $\beta_{1}<\cdots<\beta_{j}$ are elements of $A_{\alpha+1, n}$ and $y_{\beta_{l}} \in P_{n, \beta_{l}}$ for each $1 \leq l \leq j$. If $\beta_{l} \in D_{\alpha, n}$ for some $1 \leq l \leq j$, then $x_{\alpha}-y_{\beta_{l}} \in \Delta_{\alpha} \backslash P\left(n, B_{n}\right)$. Possibly replacing $x$ by $x-y_{\beta_{l}}$, we can assume that $\beta_{l} \in A_{\alpha, n}$ for all $1 \leq l \leq j$. But then $x_{\alpha} \in \Delta_{\alpha} \cap M_{\alpha} \subseteq \sum_{\beta<\alpha, \beta \in S} \Delta_{\beta}$, in contradiction with the minimality of $\alpha$.

This proves that $N$ is strong in $P$.

We can now improve the second part of Lemma 2.4:

Corollary 2.10. Let $R$ be a countable ring. Let $\mathscr{C}$ be a class of countably presented modules that has Property $(C+)$. Let $\left(P_{n} \mid n<\omega\right)$ be an increasing pure chain of modules such that $P_{n} \in \operatorname{Sum}(\mathscr{C})$ for all $n<\omega$, and let $P=\bigcup_{n<\omega} P_{n}$.

Then $P$ is the union of a continuous increasing pure chain $\mathcal{N}=\left(N_{\alpha} \mid \alpha<\aleph_{1}\right)$ consisting of strong submodules of $P$ such that $N_{\alpha+1} / N_{\alpha} \in \operatorname{Sum}(\mathscr{C})$ for all $\alpha<\aleph_{1}$.

Proof. Let $\mathcal{M}$ be the chain constructed in the second part of Lemma 2.4, and consider the corresponding family $\mathscr{H}$, as in Theorem 2.9. By [Štovíček 2012], one can select from $\mathscr{H}$ an increasing continuous chain $\mathcal{N}=\left(N_{\alpha} \mid \alpha<\aleph_{1}\right)$ of length no greater than $\aleph_{1}$, such that $N_{\alpha+1} / N_{\alpha}$ is isomorphic to a direct sum of some of the successive factors of the original chain $\mathcal{M}$, for all $\alpha<\aleph_{1}$. By Lemma 2.4, all these factors are in Sum $(\mathscr{C})$. Since $\mathscr{H}$ consists of strong (and hence pure) submodules of $P$, so does $\mathcal{N}$. 


\section{The failure of Condition (P)}

In this section we prove that Condition $(\mathrm{P})$ fails for Prüfer domains of finite character with uncountable spectrum, and, notably, for every principal ideal domain with an uncountable spectrum. We adopt [Eklof and Mekler 2002, Theorem VII.1.4] to illustrate that failure of Condition $(\mathrm{P})$ has little, if any, restriction on the $\Gamma$-invariant of even large almost-projective modules.

Recall from [Fuchs and Salce 2001, Chapter III, Lemma 2.7] that in a Prüfer domain of finite character, every maximal ideal contains a finitely generated ideal, which is not contained in any other maximal ideal. Selecting one for every maximal ideal, we obtain a system of pairwise coprime proper invertible ideals. In fact, all we need is such a system of ideals:

Theorem 3.1. Let $R$ be a commutative domain with uncountably many pairwise coprime invertible proper ideals. Let $\kappa$ be a regular uncountable cardinal, and $E$ be a nonreflecting stationary subset of $\kappa$, all of whose elements have cofinality $\omega$. Then there is a $\kappa$-projective $\kappa$-generated $R$-module $M$ with $\Gamma$-invariant $\widetilde{E}$ that is a union of a countable pure chain of projective submodules.

Before proving Theorem 3.1, we follow the suggestion of the referee and present a simple particular case of the construction.

Example 3.2. Let $R$ be a principal ideal domain with uncountably many maximal ideals $\left(p_{\alpha}\right)$ for $0<\alpha<\aleph_{1}$.

We define our module via generators and relations:

$$
P:=\left\langle e_{\alpha, n}: \alpha<\omega_{1}, n<\omega \mid p_{\alpha} e_{\alpha, n+1}=e_{\alpha, n}+e_{0, n+1}: \alpha>0\right\rangle .
$$

(This is an example for the theorem with $\kappa=\aleph_{1}$, and $E=\left\{\alpha<\aleph_{1} \mid \operatorname{cf}(\alpha)=\aleph_{0}\right\}$.)

We leave it to the reader to verify that for every $0<\alpha \leq \aleph_{1}$ and $i<\omega$, the submodule

$$
N_{\alpha, i}=\left\langle e_{\beta, j}: j \leq i, \beta<\alpha\right\rangle
$$

is actually free, with a basis formed by the $e_{0, j}$ for $j \leq i$ and the $e_{\beta, i}$ for $0<\beta<\alpha$. Since

$$
N_{\alpha, i+1} / N_{\alpha, i} \cong\left\langle R, p_{\beta}^{-1}: 0<\beta<\alpha\right\rangle
$$

(with $e_{0, i+1}$ corresponding to 1 and $e_{\beta, i+1}$ corresponding to $p_{\beta}^{-1}$ ) is torsion-free, $N_{\alpha, i}$ is a pure submodule of $N_{\alpha, i+1}$. Hence, $P$ is a union of a pure chain $P_{i}=N_{\aleph_{1}, i}$ of projective submodules.

On the other hand, $P$ is a union of a continuous chain

$$
N_{\alpha}=\bigcup_{i<\omega} N_{\alpha, i}=\left\langle e_{\beta, i}: \beta<\alpha, i<\omega\right\rangle, \quad 0<\alpha<\aleph_{1}
$$


of (strong) submodules with nonprojective factors $N_{\alpha+1} / N_{\alpha} \cong R\left[p_{\alpha}^{-1}\right]$ (with $e_{\alpha, i}$ corresponding to $p_{\alpha}^{-i}$ ), and hence is not projective.

The proof of Theorem 3.1 is mostly the same as that of [Eklof and Mekler 2002, Theorems VII.1.3-4], so we present only the differences. To include the sequence of submodules in the structure, we work in the category of $\omega$-filtered modules, that is, modules $M$ together with an increasing sequence $(M(n): n<\omega)$ of submodules satisfying $\bigcup_{n=0}^{\infty} M(n)=M$. A filtered submodule of $M$ is a submodule $N$ together with the filtration $N(n):=M(n) \cap N$. Note that $M / N$ is also a filtered module, with the filtration $(M(n) / N(n) \cong(M(n)+N) / N: n<\omega)$.

For the free module $R^{(\lambda \times \omega)}$, we always use the filtration $\left(R^{(\lambda \times n)}: n<\omega\right)$.

For a module $N$, let $N[n]$ denote the filtered module

$$
N[n](m):= \begin{cases}0, & m<n, \\ N, & m \geq n .\end{cases}
$$

For example, $R^{(\lambda \times \omega)}=\bigoplus_{n=0}^{\infty} R^{(\lambda)}[n+1]$ as filtered modules.

Proof of Theorem 3.1. We distinguish the cases $\kappa>\aleph_{1}$ and $\kappa=\aleph_{1}$. To avoid repetition, we first provide the common part of both cases, and then fill out the missing parts separately.

We build a continuous increasing chain of $\omega$-filtered modules $\left(M_{\mu}: \mu<\kappa\right)$ whose filtrations consist of pure and projective submodules. By "increasing", we mean that $M_{\nu}$ is a filtered submodule of $M_{\mu}$ for $\mu<v$.

The union $M$ of the chain is our $\kappa$-projective module with $\Gamma$-invariant $\widetilde{E}$.

To ensure that all the $M_{\mu}(n)$ and $M(n)$ are projective, we make the filtrations of the $M_{\mu+1} / M_{\mu}$ consist of projective modules.

We fix an infinite cardinal $\lambda<\kappa$. For $\mu \notin E$, let

$$
M_{\mu+1}:=M_{\mu} \oplus P_{\mu}, \quad P_{\mu}:=R^{(\lambda \times \omega)}=\bigoplus_{n=0}^{\infty} R^{(\lambda)}[n+1] e_{\mu, n} .
$$

For the case $\mu \in E$, we select a template as in [Eklof and Mekler 2002, Corollary VII.1.2], that is, a nonprojective $\lambda$-generated module $N_{\mu}$ with an $\omega$-filtration by projective modules. By adding a projective module, we may assume that the filtration consists of $\lambda$-generated free modules; that is, $N_{\mu}(n) \cong R^{(\lambda)}$. The filtration induces a short exact sequence

$$
0 \rightarrow K_{\mu} \rightarrow F_{\mu} \rightarrow N_{\mu} \rightarrow 0
$$

of $\omega$-filtered modules, where

$$
F_{\mu}:=\bigoplus_{n=0}^{\infty} N_{\mu}(n)[n] e_{n}, \quad K_{\mu}:=\bigoplus_{n=0}^{\infty} N_{\mu}(n)[n+1] e_{n} \cong R^{(\lambda \times \omega)} .
$$


The embedding of $K_{\mu}$ into $F_{\mu}$ maps $x e_{n}$ into $x e_{n+1}-x e_{n}$, and the homomorphism $F_{\mu} \rightarrow N_{\mu}$ maps $x e_{n}$ into $x$ for any $x \in N_{\mu}(n)$ and natural number $n$. In particular, the filtrations of $K_{\mu}$ and $F_{\mu}$ consist of direct summands, and hence of pure and projective submodules. We see that the modules

$$
F_{\mu} /\left(K_{\mu}(n)\right)=\bigoplus_{m=n}^{\infty} N_{\mu}(m)
$$

are projective for all $N$.

We define $M_{\mu+1}$ as the pushout of the inclusion $K_{\mu} \subseteq F_{\mu}$ by a suitable embedding $K_{\mu} \rightarrow M_{\mu}$ identifying $K_{\mu}$ with the direct summand

$$
\bigoplus_{n=0}^{\infty} R^{(\lambda)}[n+1] e_{\mu_{n}, n}
$$

of the filtered submodule $\bigoplus_{n=0}^{\infty} P_{\mu_{n}}$ for an increasing sequence of successor ordinals $\mu_{n}$ with supremum $\mu$. Then

$$
M_{\mu+1} / M_{\mu} \cong N_{\mu}
$$

as filtered modules, and therefore $M_{\mu+1} / M_{\mu}$ is filtered by projective submodules.

The rest of [Eklof and Mekler 2002, Theorems VII.1.3-4] apply to show that $M$ is a $\kappa$-free module of $\Gamma$-invariant $\widetilde{E}$. The filtration of $M$ consists of projective submodules by construction.

All that is left is to find $\lambda$ and the $N_{\mu}$ and to verify that the filtration of $M$ actually consists of pure submodules.

When $\kappa>\aleph_{1}$, we choose $\lambda=\aleph_{1}$, and let $N_{\mu}$ be an $\aleph_{1}$-generated nonprojective module with an $\omega$-filtration by pure and projective submodules. (We may choose all the $N_{\mu}$ the same.) Such an $N_{\mu}$ exists by the $\kappa=\aleph_{1}$ case. Since the filtration of $N_{\mu}$ is by pure submodules, it follows that all the $M_{\mu}(n)$ and $M(n)$ are pure submodules.

When $\kappa=\aleph_{1}$, we let $\lambda=\aleph_{0}$. Let $\left(I_{\alpha}: \alpha<\aleph_{1}\right)$ be a collection of pairwise coprime invertible proper ideals of $R$. We define the $N_{\mu}$ as submodules of the quotient field of $R$ :

$$
N_{\mu}(n):=I_{\mu}^{-n}, \quad N_{\mu}:=I_{\mu}^{-\infty} .
$$

Clearly, $N_{\mu}$ is nonprojective and its filtration is by projective submodules.

To show that the filtration of the $M_{\mu}$ is pure, we show that its localization by any maximal ideal $Q$ is pure. When $I_{\mu} \nsubseteq Q$ and $\mu \in E$, then $N_{\mu, Q}=R_{Q}[0]$, so the short exact sequence $K_{\mu, Q} \rightarrow F_{\mu, Q} \rightarrow N_{\mu, Q}$ of filtered modules splits, and hence $M_{\mu+1, Q}=M_{\mu, Q} \oplus N_{\mu, Q}$ as filtered modules. 
There is at most one $\mu \in E$ with $I_{\mu} \subseteq Q$. Hence, by the previous paragraph, if there is such a $\mu$, then $M_{\nu, Q}$ is a direct summand of $M_{\nu+1, Q}$ as filtered modules, for all $v<\mu$. So

$$
M_{\mu, Q} \cong \bigoplus_{\nu<\mu} M_{\nu+1, Q} / M_{\nu, Q},
$$

with an arbitrary choice of split preimages of the $M_{\nu+1, Q} / M_{\nu, Q}$. Recall that $K_{\mu, Q}$ is a direct summand of a sum of some of these preimages, so it is actually a direct summand of $M_{\mu, Q}$; that is, $M_{\mu, Q}=K_{\mu, Q} \oplus H_{\mu}$. It follows that

$$
M_{\mu+1, Q}=F_{\mu, Q} \oplus H_{\mu} .
$$

These decompositions of filtered modules show that the filtrations of $H_{\mu}$ and $M_{\mu+1, Q}$ consist of pure submodules.

We finish with:

Problem 3.3. Characterize the rings $R$ satisfying Condition (P).

\section{References}

[Drinfeld 2006] V. Drinfeld, "Infinite-dimensional vector bundles in algebraic geometry: an introduction", pp. 263-304 in The unity of mathematics: in honor of the nintieth birthday of I. M. Gelfand, edited by P. I. Etingof et al., Progr. Math. 244, Birkhäuser, Boston, MA, 2006. MR 2007d:14038 Zbl 1108.14012

[Eklof 1993] P. C. Eklof, "Set-theoretic methods: the uses of gamma invariants", pp. 43-53 in Abelian groups (Curaçao, 1991), edited by L. Fuchs and R. Gobel, Lecture Notes in Pure and Appl. Math. 146, Dekker, New York, 1993. MR 94d:20069 Zbl 0812.20033

[Eklof and Mekler 2002] P. C. Eklof and A. H. Mekler, Almost free modules, North-Holland Mathematical Library 65, North-Holland, Amsterdam, 2002. MR 2003e:20002 Zbl 1054.20037

[Fuchs and Rangaswamy 2011] L. Fuchs and K. M. Rangaswamy, "Chains of projective modules", J. Algebra Appl. 10:1 (2011), 167-180. MR 2784760 Zbl 1213.13024

[Fuchs and Salce 2001] L. Fuchs and L. Salce, Modules over non-Noetherian domains, Mathematical Surveys and Monographs 84, Amer. Math. Soc., Providence, RI, 2001. MR 2001i:13002 Zbl 0973.13001

[Göbel and Trlifaj 2006] R. Göbel and J. Trlifaj, Approximations and endomorphism algebras of modules, de Gruyter Expositions in Mathematics 41, de Gruyter, Berlin, 2006. MR 2007m:16007 Zbl 1121.16002

[Herbera and Trlifaj 2009] D. Herbera and J. Trlifaj, "Almost free modules and Mittag-Leffler conditions", preprint, 2009. To appear in Adv. Math. arXiv 0910.4277

[Hill 1970] P. Hill, "On the freeness of abelian groups: A generalization of Pontryagin's theorem", Bull. Amer. Math. Soc. 76 (1970), 1118-1120. MR 41 \#8518 Zbl 0223.20058

[Macías-Díaz 2010] J. Macías-Díaz, "A generalization of the Pontryagin-Hill theorems to projective modules over Prüfer domains", Pacific J. Math. 246:2 (2010), 391-405. MR 2011i:13010 Zbl 1205.13014

[Raynaud and Gruson 1971] M. Raynaud and L. Gruson, "Critères de platitude et de projectivité", Invent. Math. 13 (1971), 1-89. MR 46 \#7219 Zbl 0227.14010 
[Rothmaler 1994] P. Rothmaler, Mittag-Leffler modules and positive atomicity, Habilitationsschrift, Christian-Albrechts-Universität zu Kiel, 1994.

[Rothmaler 1997] P. Rothmaler, "Mittag-Leffler modules", Ann. Pure Appl. Logic 88:2-3 (1997), 227-239. MR 99b:16006 Zbl 0916.16004

[Š́ovíček 2012] J. Š́ovíček, "Deconstructibility and the Hill lemma in Grothendieck categories", (2012). To appear in Forum Math., DOI: 10.1515/FORM.2011.113.

[Trlifaj 1995] J. Trlifaj, "Strong incompactness for some nonperfect rings", Proc. Amer. Math. Soc. 123:1 (1995), 21-25. MR 95c:16003 Zbl 0827.16001

[Trlifaj 1996] J. Trlifaj, "Whitehead test modules”, Trans. Amer. Math. Soc. 348:4 (1996), 15211554. MR 96h:16008 Zbl 0865.16006

Received May 13, 2011. Revised September 12, 2011.

GÁBOR BRAUN

INSTITUT FÜR INFORMATIK

UNIVERSITÄT LEIPZIG

PF 100920

D-04009 LEIPZIG

GERMANY

gabor.braun@uni-duisburg-essen.de

JAN TRLIFAJ

DEPARTMENT OF AlgEBRA

Charles University, FaCUlty of Mathematics AND Physics

SOKOLOVSKÁ 83

18675 PRAGUE 8

CZECH RePublic

trlifaj@karlin.mff.cuni.cz 


\title{
PACIFIC JOURNAL OF MATHEMATICS
}

\author{
http://pacificmath.org \\ Founded in 1951 by \\ E. F. Beckenbach (1906-1982) and F. Wolf (1904-1989)
}

\section{EDITORS}

V. S. Varadarajan (Managing Editor)

Department of Mathematics

University of California

Los Angeles, CA 90095-1555

pacific@math.ucla.edu

Vyjayanthi Chari

Department of Mathematics

University of California

Riverside, CA 92521-0135

chari@math.ucr.edu

\section{Robert Finn}

Department of Mathematics Stanford University

Stanford, CA 94305-2125

finn@math.stanford.edu

Kefeng Liu

Department of Mathematics

University of California

Los Angeles, CA 90095-1555

liu@math.ucla.edu
Darren Long

Department of Mathematics

University of California

Santa Barbara, CA 93106-3080

long@math.ucsb.edu

Jiang-Hua Lu

Department of Mathematics

The University of Hong Kong

Pokfulam Rd., Hong Kong jhlu@maths.hku.hk

Alexander Merkurjev

Department of Mathematics

University of California

Los Angeles, CA 90095-1555

merkurev@math.ucla.edu
Sorin Popa

Department of Mathematics University of California

Los Angeles, CA 90095-1555 popa@math.ucla.edu

Jie Qing

Department of Mathematics

University of California

Santa Cruz, CA 95064

qing@cats.ucsc.edu

Jonathan Rogawski

Department of Mathematics

University of California

Los Angeles, CA 90095-1555

jonr@math.ucla.edu

\section{PRODUCTION}

pacific@math.berkeley.edu

\section{SUPPORTING INSTITUTIONS}

ACADEMIA SINICA, TAIPEI

CALIFORNIA INST. OF TECHNOLOGY INST. DE MATEMÁTICA PURA E APLICADA KEIO UNIVERSITY

MATH. SCIENCES RESEARCH INSTITUTE NEW MEXICO STATE UNIV.

OREGON STATE UNIV.

\author{
STANFORD UNIVERSITY \\ UNIV. OF BRITISH COLUMBIA \\ UNIV. OF CALIFORNIA, BERKELEY \\ UNIV. OF CALIFORNIA, DAVIS \\ UNIV. OF CALIFORNIA, LOS ANGELES \\ UNIV. OF CALIFORNIA, RIVERSIDE \\ UNIV. OF CALIFORNIA, SAN DIEGO \\ UNIV. OF CALIF., SANTA BARBARA
}

\author{
UNIV. OF CALIF., SANTA CRUZ \\ UNIV. OF MONTANA \\ UNIV. OF OREGON \\ UNIV. OF SOUTHERN CALIFORNIA \\ UNIV. OF UTAH \\ UNIV. OF WASHINGTON \\ WASHINGTON STATE UNIVERSITY
}

These supporting institutions contribute to the cost of publication of this Journal, but they are not owners or publishers and have no responsibility for its contents or policies.

See inside back cover or pacificmath.org for submission instructions.

The subscription price for 2011 is US \$420/year for the electronic version, and \$485/year for print and electronic.

Subscriptions, requests for back issues from the last three years and changes of subscribers address should be sent to Pacific Journal of Mathematics, P.O. Box 4163, Berkeley, CA 94704-0163, U.S.A. Prior back issues are obtainable from Periodicals Service Company, 11 Main Street, Germantown, NY 12526-5635. The Pacific Journal of Mathematics is indexed by Mathematical Reviews, Zentralblatt MATH, PASCAL CNRS Index, Referativnyi Zhurnal, Current Mathematical Publications and the Science Citation Index.

The Pacific Journal of Mathematics (ISSN 0030-8730) at the University of California, c/o Department of Mathematics, 969 Evans Hall, Berkeley, CA 94720-3840, is published monthly except July and August. Periodical rate postage paid at Berkeley, CA 94704, and additional mailing offices. POSTMASTER: send address changes to Pacific Journal of Mathematics, P.O. Box 4163, Berkeley, CA 94704-0163.

PJM peer review and production are managed by EditFLOW ${ }^{\mathrm{TM}}$ from Mathematical Sciences Publishers.

PUBLISHED BY PACIFIC JOURNAL OF MATHEMATICS

at the University of California, Berkeley 94720-3840

A NON-PROFIT CORPORATION

Typeset in LATEX

Copyright $(2011$ by Pacific Journal of Mathematics 


\section{PACIFIC JOURNAL OF MATHEMATICS}

Volume $254 \quad$ No. $1 \quad$ November 2011

A mean curvature estimate for cylindrically bounded submanifolds

Luis J. AlíAS and MARCos DAJCZER

Weyl group multiple Dirichlet series of type $C$

JENNIFER BEINEKE, BENJAMIN BRUBAKER and SHARON FrECHETTE

Milnor open books of links of some rational surface singularities

MOHAN BHUPAL and BURAK OZBAGCI

Simple closed curves, word length, and nilpotent quotients of free groups

KHALID BOU-RABEE and ASAF HADARI

Strong submodules of almost projective modules

GÁBOR BRAUN and JAN TRLIFAJ

Interlacing log-concavity of the Boros-Moll polynomials

William Y. C. Chen, LARry X. W. WAng and ERnest X. W. Xia

Schwarzian norms and two-point distortion

Martin Chuaqui, Peter Duren, William Ma, Diego Mejía, David

MINDA and BRAD OSGOOD

The principle of stationary phase for the Fourier transform of $D$-modules

JIANGXUE FANG

Monotonicity and uniqueness of a 3D transonic shock solution in a conic nozzle with

variable end pressure

JUN LI, ZHOUPING XIN and HUICHENG YIN

Refined open noncommutative Donaldson-Thomas invariants for small crepant resolutions

KENTARO NAGAO

The Dirichlet problem for harmonic functions on compact sets

TONY L. PERKINS

Extension of an analytic disc and domains in $\mathbb{C}^{2}$ with noncompact automorphism group

\section{MINJU SONG}

Regularity of the first eigenvalue of the $p$-Laplacian and Yamabe invariant along geometric flows

ER-MIN WANG and YU ZHENG 